\title{
Sken\&agraphie
}

skilingraphil Coulisses des arts du spectacle et des scènes

émergentes

$5 \mid 2018$

Juste la fin du monde, de Lagarce à Dolan

\section{Juste la fin du mot : l'hybridité de la parole déconstruite dans le théâtre de Jean-Luc Lagarce}

\section{Alina Kornienko}

\section{OpenEdition}

\section{Journals}

Édition électronique

URL : http://journals.openedition.org/skenegraphie/1398

DOI : 10.4000/skenegraphie.1398

ISSN : 2553-1875

\section{Éditeur}

Presses universitaires de Franche-Comté

\section{Édition imprimée}

Date de publication : 1 janvier 2018

Pagination : $71-80$

ISBN : 978-2-84867-5609-8

ISSN : $1150-594 X$

Référence électronique

Alina Kornienko, « Juste la fin du mot: l'hybridité de la parole déconstruite dans le théâtre de Jean-Luc Lagarce », Sken\&agraphie [En ligne], 5 | 2018, mis en ligne le 01 janvier 2019, consulté le 20 avril 2019. URL : http://journals.openedition.org/skenegraphie/1398; DOI : 10.4000/skenegraphie.1398

Ce document a été généré automatiquement le 20 avril 2019

Presses universitaires de Franche-Comté 


\title{
Juste la fin du mot : l'hybridité de la parole déconstruite dans le théâtre de Jean-Luc Lagarce
}

\author{
Alina Kornienko
}

Je vais m'atteler très vite à une pièce. Une pièce courte qui me trottait dans la tête depuis quelque

temps.

Cela s'appelle Les Adieux.

Cinq personnages, la mère, le père, la sœur, le fils et l'ami du fils.

Le fils vient, revient. Il va mourir, il est encore jeune. Il n'a jamais vraiment parlé. Il vient écouter. Il est avec un homme. Ils passent une journée là à ne pas faire grand-chose. Ils écoutent. La mère parle tout le temps. Éviter le silence, faire comme si de rien n'était.

On ne le dit pas, mais on sait que l'on ne se reverra

jamais. ${ }^{1}$

1 L'héritage littéraire de Jean-Luc Lagarce propose un univers au sein duquel il existe un lien indissoluble entre toutes ses œuvres, fait notamment de rapports intertextuels. Une telle force créatrice engage une hybridité qui est à l'œuvre aussi bien à l'échelle micro que macroscopique et englobe perspectives générique, poétique et esthétique. La note introductrice de cet article, datée de "jeudi 11 février 1988 » dans le premier tome du Journal de Lagarce contient le projet d'une pièce, Les Adieux (reprise modifiée de son projet de roman homonyme), qui n'est en réalité, que la toute première esquisse du Pays lointain dont l'écriture aurait lieu sept ans plus tard, même si elle inspire une première version intitulée Juste la fin du monde deux ans plus tard. Ce synopsis que propose Lagarce dans le cadre d'un récit autobiographique intime est moins un projet dramatiquement structuré qu'un cadre émotionnel. Cette esquisse raconte d'une manière chaotique et abrupte la fable de la pièce en mettant en valeur les troubles communicatifs de la future pièce. Cette 
étape d'éclosion de la conception créatrice donne à voir les premiers pas que fait l'écrivain en concevant son nouveau texte. L'insertion de cet extrait dans l'ensemble du texte du Journal est assurée notamment grâce à l'émotion et à la spontanéité : celle-ci entre dans la logique du texte intime, écrit en n'importe quel lieu, qui présente toute sorte de pensées et de sentiments de son auteur. La note en question montre que le Journal agissait comme une réserve d'idées artistiques susceptible de servir de source d'inspiration.

2 La pièce Juste la fin du monde (1990) fait partie du cycle dramatique qu'on peut dire du " fils prodigue », composé de quatre pièces écrites entre 1984 et 1995, parmi lesquelles Retour à la citadelle (1984), J'étais dans ma maison et j'attendais que la pluie vienne (1994) et Le Pays lointain (1995). Si l'on revient au synopsis noté dans le Journal, préfigurant directement Le Pays lointain, Juste la fin du monde paraît beaucoup plus simple : moins de personnages - absence de la figure de « l'ami du fils » - et moins d'effet d'antithèse entre les deux familles de Louis. Le Père sera également une figure absente de Juste la fin $d u$ monde, alors qu'il revient comme personnage transgressif fantomatique dans Le Pays lointain. Il convient de noter que le frère de Louis, Antoine, avec sa femme Catherine sont complètement absents dans la toute première conception de cette fable dramatique alors qu'ils joueront un rôle très important dans les deux pièces en question. Dans son Journal, Lagarce mentionne à plusieurs reprises les problèmes de communication avec ses proches. Cela ne nous donne pourtant pas le droit de postuler que sa dernière pièce, ainsi que tout le cycle du "fils prodigue", soit une sorte de message d'adieu qui leur serait adressé. Il est évident que chaque œuvre littéraire est affectée par l'expérience personnelle de celui qui l'écrit; en revanche, son ambition excède le cadre d'une simple transcription autobiographique de cette expérience grâce à son caractère empirique qui implique une distinction stricte entre le «je» auctorial et le «je» fictionnel du protagoniste. On pourrait dire que Louis est un personnage lyrique propre au romantisme dont le développement est assuré par le cycle dramatique du «fils prodigue » dans son intégralité. Car, d'après sa définition, le personnage lyrique reflète l'expérience morale, éthique et existentielle de l'auteur, lui sert d'instrument littéraire d'auto-observation sans pour autant être un alter-ego auctorial. Le rapport de l'auteur à son personnage lyrique est le même que dans tous les autres cas d'invention poétique. Il est également notoire que les expressions comme "retour à la citadelle» ou «le pays lointain», devenues ensuite les titres des œuvres du cycle dramatique en question, se répètent tout au long du corpus textuel intégral du Journal de Lagarce dans le contexte de la description de ses relations familiales ou du milieu originel.

3 Au sein de son univers dramatique et littéraire, Lagarce transgresse les données initiales : le Prologue qui ouvre l'action scénique de Juste la fin du monde dans une longue réplique monologale de Louis se transforme, dans Le Pays lointain, en un dialogue avec ses personnages alter-ego Longue Date et L'Amant, mort déjà. Cette extériorisation du dialogue interne avec soi trouve son origine dans la «Tentative de réécriture. Seconde version » (non datée) de Juste la fin du monde ${ }^{2}$. Cette version de la pièce en question s'ouvre par un Prologue totalement transformé en un dialogue entre Louis et Longue Date. Il faut à nouveau revenir au projet de la pièce Les Adieux cité auparavant où Lagarce annonce parmi les cinq personnages l'ami du fils. Cela met encore une fois en évidence un point de convergence sous-jacent entre tous les textes de Lagarce. Il est également notoire dans cette perspective que l'implication d'un " personnage-miroir » n'est pas encore intégrale car Longue Date ne paraît pas dans chaque séquence. Le canevas dramatique reste encore 
très proche des précédentes versions de Juste la fin du monde. Longue Date, tout en étant encore placé de côte du dialogue dramatique principal, ne parle qu'avec Louis et Le Père une figure complétement absente dans Juste la fin du monde et fantomatique dans Le Pays lointain. Il est évident dans cette perspective que l'intimité du cercle familial mise en scène dans Juste la fin du monde est à la fois paradoxalement brisée et renforcée par l'introduction de ces personnages dans Le Pays lointain. Ceux-ci permettent de constituer une partie introductive d'initiation et de présentation beaucoup plus longue que dans Juste la fin du monde. Il faut aussi mentionner que dans cette même "tentative de réécriture » la division en scènes numérotées successives de Juste la fin du monde est modifiée au profit d'une division en séquences-scènes non-numérotées. L'Épilogue, constitué d'un monologue de Louis qui se fonde sur un souvenir personnel décrit par Lagarce dans son Journal, est absolument identique à la « séquence » / scène finale du Pays lointain.

Le conflit mis en scène dans Juste la fin du monde (ainsi que dans Le Pays lointain) repose sur une problématique relative à la communication. En effet, l'action dramatique se construit autour du retour du personnage principal, Louis, qui revient, après des années d'absence, voir sa famille pour la dernière fois et leur annoncer sa mort prochaine. Il essaie de réinstaurer le dialogue avec sa famille et il n'y parvient pas, sans même réussir à éviter d'accroître le marais d'incompréhension réciproque, de mensonges et de vaines promesses. Nous le percevons facilement dans les hésitations des personnages, qui créent une continuité entre les situations du présent et du passé ( $«$ je crois, nous croyons, nous avons cru, je crois que c'est bien $\left.»^{3}\right)$. Tout au long de l'action dramatique qui n'est en réalité qu'une décomposition du dialogue entre les locuteurs, les personnages se trouvent en quête infinie du mot juste. Les répliques sont inondées de clichés de communication. Ainsi, au début de la pièce, Catherine tout en parlant à Louis de ses enfants demande sans cesse si ce sujet de conversation n'ennuie pas son locuteur. La tentative de restauration du dialogue antérieur, ainsi que les relations compliquées d'incompréhension entre les interlocuteurs, renforcent encore ce recours abusif aux clichés : les membres de la famille semblent se perdre lors de la scène initiale de la première partie : la question banale et presque inutile dans cette situation communicative entre "tutoyer" ou "vouvoyer" devient pour eux cruciale et existentielle. Cela fait naître un formalisme communicatif entre les membres de ce cercle dialogal censé être intime. La matière poétique à la fois très hétérogène et très réaliste de Juste la fin $d u$ monde est tissée notamment par la transcription d'un sociolecte de la petite bourgeoisie française. «Comment est-ce que tu as dit? Une "recommandation" que tu t'es fait, faite ? merde ${ }^{4} »$ : ce pur échec langagier démontre que le langage fonctionne comme une institution sociale. Lagarce se sert très souvent de cette hypercorrection de la langue, abusive, parfois fausse et toujours absurde alourdissant la communication dans le milieu de la petite bourgeoisie. Les dialogues manquent de cohérence ; la division en scènes du texte dramatique, structuration assez rare au sein de l'œuvre complète de Lagarce, est aussi plutôt formelle ; par exemple, entre la première et la seconde scène, il n'y a pas de changement clair dans la situation dramatique. Les personnages semblent se perdre dans leur propre discours parmi les multiples incises, au milieu de phrases longues faisant souvent obstacle à la compréhension écrite ou orale. Il est également très significatif que ce trait de la poétique dramatique de Lagarce soit présent dès les toutes premières versions de l'ouvrage. Suzanne semble se perdre dans les incises de son monologue qui compose toute la troisième scène de Juste la fin du monde : «Elle, ta mère, ma mère, elle $[. ..] »^{5}$. Comme tous les personnages de Lagarce, elle respecte de manière obsessionnelle les normes sociales et 
communicatives tout en laissant de côté - et excluant donc - le message communicatif même. Leur discours, ainsi que chaque mot qu'ils corrigent et essayent d'employer au plus près, se dévide du sens. Nous faisons face, par conséquent, au paradoxe sur lequel se construit l'univers dramatique de Jean-Luc Lagarce: cette recherche infinie d'un mot juste mène à son dévidement et à son autodestruction. Pourtant, le langage est toujours au centre de la dramaturgie en question. Il est son moteur le plus significatif. En comparant la première version et les suivantes de Juste la fin $\mathrm{du}$ monde, nous voyons clairement que, par exemple, au sein de ce même monologue de Suzanne, Lagarce souligne les incises avec une ponctuation d'attaque communicative - le tiret - de la main, après avoir imprimé le texte intégral. Tout en parlant de ces incises il faut noter que la "tentative de réécriture" de Juste la fin du monde présente parfois des séries d'associations plus radicales et même violentes d'un point de vue communicatif que la rédaction finale du texte (voir le monologue de Suzanne et «notre bien chère mère »). Dans le Prologue, Louis, à la fin de son monologue introductif, affirme sa volonté d'être « [s]on propre maitre $»^{6}$ ce qui signifie aussi maître de sa parole. La fable dramatique, qui débute par une annonce de l'acte de parole, présente le drame du non-dit, le drame du langage. Ce paradoxe est constitutif d'un univers dramatique au sein duquel l'acte de parole devient un acte créateur :

Le récit de toutes ces années, l'histoire sans histoire d'un homme dans la France de ces vingt dernières années, les rencontres, la famille, les amis, les amours rencontrées et vécues, le travail et les aventures ${ }^{7}$.

5 Juste la fin du monde décrit soigneusement, comme Le Pays lointain, l'homme français ordinaire, contemporain, ainsi que toute sa vie quotidienne lors des dernières décennies $\mathrm{du} \mathrm{Xx}^{\mathrm{e}}$ siècle. Ces deux textes exposent et incarnent la génération à laquelle appartenait Lagarce. C'est la raison pour laquelle son « fils prodigue » n'est pas un pécheur qui revient dans sa maison natale pour faire part de son échec et commencer une nouvelle vie; il est celui qu'on ne comprend pas encore mais qui n'est pas seul dans la société (si l'on prend en compte le début de la série des coming-out dans la société française de certaines personnes publiques et respectées à l'époque). C'est un messager censé apporter l'ultime nouvelle qui revient et salue sa famille pour toujours. Il veut avouer, s'avouer, mais pas pour changer ou se changer; il aimerait le faire pour partager, en suivant, d'une certaine manière, la tradition et la norme sociale, même si celle-ci lui est encore hostile et étrangère. Le drame du retour mise en scène par Jean-Luc Lagarce est, d'une certaine manière, beaucoup plus un drame du retour de la parole et sur la parole qu'un drame du retour d'un fils dans sa famille, avec ses relations tendues et un dialogue difficile, ce qui donne son caractère métadramatique au cycle entier du « fils prodigue ».

6 Le «fils prodigue » de Lagarce et surtout son personnage de Louis incarnent de manière emblématique le prototype contemporain de l'homme de communication - et de noncommunication. Tout commence par le fait qu'il écrit et ne téléphone pas à ses proches. Suzanne, elle aussi, par exemple, dans son soliloque ${ }^{8}$ au début de Juste la fin du monde, constate que l'importance donnée à un individu passe par la quantité de mots, écrits et parlés, qu'on lui adresse. Louis revient afin de revoir sa famille et n'est pas capable de leur dire le message avec lequel il est venu. Le seul dialogue qu'il est capable de mener est celui avec ses alter-égo. Il est pourtant très significatif que même ce dialogue avec les différentes « versions » de soi ne soit pas fructueux. Le «fils prodigue » de Lagarce peut, par conséquent, être considéré comme la représentation dramatique et littéraire de la 
poétique de l'époque: elle se construit autour d'une parole qui ne sert plus à communiquer dans son sens classique.

7 Tout en suivant la logique générale des œuvres de Lagarce, Juste la fin du monde met en scène le conflit d'incompréhension de l'Autre ainsi que de soi. Le véritable enjeu dramatique réside donc sur le plan langagier. L'intrigue n'est qu'un prétexte pour la construction d'une dramaturgie spécifique à Lagarce. La tragédie vécue par chacun de ses personnages en général, et de son « fils prodigue » en particulier, est de ne pas pouvoir dire le mot juste: ils sont constamment en train de le chercher tout en étant dans l'incapacité d'entendre et de comprendre ce mot de la part de l'Autre. Lagarce propose ainsi une poétique de la décadence communicative et langagière à partir des principes constructifs d'un métadrame. Selon la définition de Jean-Pierre Sarrazac, le métadrame est un « drame sur un autre drame » dont « le procédé du théâtre dans le théâtre n'est qu'une modalité parmi d'autres »:

le métadrame constitue l'épilogue d'un drame [...] antérieur non écrit. [...] Quintessence dramatique, conflit mis à distance, commentaire d'un drame plus que drame vécu, le métadrame entraîne une profonde mutation dans la situation du personnage : du traditionnel personnage agissant, nous passons à un personnage passif et spectateur de lui-même, de sa propre existence considérée comme révolue ${ }^{9}$

Les œuvres dramatiques de Lagarce possèdent les traits majeurs du métadrame tout en les intégrant et en les adaptant aux règles poétiques et constructives du théâtre du langage. Comme le précise Sarrazac, le métadrame met en scène un «commentaire d'un drame ", ce qui nous renvoie à la passivité des personnages de Lagarce. Êtres de papier comme tout personnage de théâtre -, ils ne regardent pas simplement leur vie, mais ils la constituent par l'acte de parole, ils la créent tout en la regardant et la racontant. Le métadramatisme communicatif qui compose les pièces de Lagarce se construit sur une nouvelle mise en abyme liée au concept de «dialogue du second degré » de Maeterlinck. Composé des non-dits, ce dialogue est porteur d'un message intuitif qui parait s'opposer au bavardage infini dépourvu d'enjeu chez Lagarce. Mais, sous le discours a priori chaotique et privé de signifié, se cachent les vrais propos qu'il faut repérer entre les lignes. Cette mise en abyme dialogale est assurée grâce à la présence d'un autre concept de Maeterlinck : celui de troisième personnage. Ce personnage qui est, chez Maeterlinck, l'incarnation du fatum, construit le lien entre l'intrigue illusoire et la parole prononcée avec ses vraies significations infinies. Au sein de l'univers dramatique de Lagarce, le troisième personnage devient la parole même - le seul personnage actif et agissant sur scène, le seul moteur de l'action ainsi que sa seule origine. Le dialogue du second degré lie, sous la plume de Lagarce, la parole dramatique et littéraire à la vie quotidienne, mettant en relief tous les tics, vices et vertus de la société contemporaine, tout en faisant attention en premier lieu aux comportements et aux schémas communicatifs mises en œuvre. C'est là, dans cette surdité totale, dans l'inconsistance langagière que réside pour Lagarce le tragique quotidien de Maeterlinck.

9 Ce tragique emprunté et réapproprié par Lagarce commence pour lui au cœur même du langage pour générer les multiples sens, les pièges infinis de la contextualisation, déconstruisant l'échange langagier habituellement fructueux entre les individus. Le métadramatisme peut être désigné, par conséquent, comme le composant majeur du théâtre lagarcien, la logorrhée étant son incarnation la plus évidente. Le métadramatisme devient, dans le cadre de la création dramatique et littéraire de Lagarce, une des représentations constructives et poétiques d'un logo-drame. Le vrai drame est celui du 
troisième personnage précédemment évoqué, de la parole en recherche constante à la fois sur elle-même et pour l'autre. Nous pouvons postuler, par conséquent, que toutes les pièces de Lagarce - et surtout son cycle du « fils prodigue » construit sur la perspective d'un message crucial à annoncer - mettent en scène un logo-drame. Celui-ci suit la logique dramatique d'Anton Tchekhov: "Les gens ne font que dîner et cependant leur bonheur naît et leurs destins se détruisent ${ }^{10} »$.

La manie de la rectification et de l'autocorrection renvoie le lecteur / spectateur au fait que la société impose des règles d'expression verbale, de réception communicative et de comportement. Les personnages de Lagarce sont assujettis à ces règles. L'auteur souligne, grâce à ses personnages, leur relativité et leur fausseté : ce ne sont pas les personnages eux-mêmes mais c'est la société qui parle sur scène car elle est présente dans les œuvres dramatiques de Lagarce au titre de citation. Le logo-drame devient ainsi une expérience du délire qui est, contrairement à cette même pratique psychanalytique, celle du langage en tant que condition primordiale et innée de toute existence humaine. La structure logodramatique développée par Lagarce met en question la propriété de la structure dialogique de l'énonciation par le fait que le «je» est toujours en position de transcendance par rapport à "tu » selon Émile Benveniste. Cela veut dire que toute énonciation au sein d'une structure dialogale entraîne l'assomption d'une axiologie interpersonnelle où «tu » n'existe qu'au service du «je». Le logo-drame révèle les malaises communicationnels dans la société contemporaine. Il met en relief les mécanismes complexes de la perception intersubjective - à la fois la perception identitaire réflexive, qui va de soi à soi, et la perception transitive, qui va de soi à l'autre ou de l'autre à soi dans le cadre d'un acte de parole. Au sein de ce drame du langage, une instance perceptive se pose donc comme centre; elle installe autour de soi un champ de présence d'où proviennent la surdité et l'incapacité ou le refus d'entendre et de comprendre son interlocuteur. Le logo-drame se présente comme un champ de bataille dialogique où la parole lancée est en quête de sa réponse. Il accomplit l'articulation entre le sujet empirique et le sujet de l'écriture. La pratique dramatique devient, sous la plume du dramaturge, le croisement conflictuel et irréductible de la vie humaine et de l'œuvre littéraire. Car la société contemporaine avec tous ses membres ne peut pas être absente de ce théâtre ; elle y existe au titre de citation. Tout en mettant en avant la parole libre, vivante, Lagarce démontre l'étrangeté de la société contemporaine par sa poétique du ressassement, des non-dits et de la logorrhée. L'étrangeté de son écriture rend compte de la poétique particulière de son époque ainsi que de son inconsistance communicative. Lagarce psychologise donc, d'une certaine manière, la temporalité poétique qu'il met en œuvre, le langage que parlent ses contemporains sans jamais psychologiser ses personnages dramatiques. En effet, il existe une distinction forte entre la psychologie et la littérature même si le théâtre psychologique peut offrir les ressorts d'un logo-drame.

Un sujet hybride se trouve exposé en un langage hybride qui se (dé)construit et se parodie. L'événement-créateur s'incarne, au sein de la dramaturgie de Jean-Luc Lagarce, en acte de parole. Juste la fin du monde devient ainsi un drame de la communication, d'un tout dernier mot - ou cri - qui ne sera jamais lancé. 


\section{NOTES}

1. Jean-LuC LAGARCE, Journal, 1977-1990, tome II, Besançon, Les Solitaires intempestifs, 2007, p. 320.

2. Texte consultable à l'adresse: http://fanum.univ-fcomte.fr/lagarce/index.php? $\mathrm{f}=1 \&$ path=_fo190_fo2_fo113_d408_d16

3. Jean-Luc LAgARCE, Juste la fin du monde, Besançon, Les Solitaires intempestifs, «Classiques contemporains », 2012, p. 34.

4. Ibid., p. 75.

5. Ibid., p. 39.

6. Ibid., p. 24.

7. Synopsis du Pays lointain consultable à l'adresse: http://fanum.univ-fcomte.fr/lagarce/ index.php? $\mathrm{f}=1 \& \mathrm{~d}=419$

8. Jean-Luc LAGARCE, Juste la fin du monde, op. cit., p. 36-43.

9. Jean-Pierre SARRAZAC (dir.), Poétique du drame moderne et contemporain. Lexique d'une recherche, Études théâtrales, n²2, 2001, p. 65-67.

10. Teatr $i$ iskusstvo, $\mathrm{n}^{\circ} 28,1904$, p. 521

\section{INDEX}

Mots-clés : Jean-Luc Lagarce, Juste la fin du monde, Le Pays lointain, Les Adieux

\section{AUTEURS}

\section{ALINA KORNIENKO}

Alina Kornienko est doctorante-boursière de l'ED Pratiques et théories du sens à l'Université ParisVIII-Vincennes-Saint-Denis. Elle est spécialiste de dramaturgie française contemporaine (l'œuvre de Jean-Luc Lagarce) et étudie notamment le statut du langage dans le théâtre contemporain 